\title{
Slipping detection and control in gripping fruits and vegetables for agricultural robot
}

\author{
Guangzhao Tian, Jun Zhou*, Baoxing Gu \\ (College of Engineering, Nanjing Agricultural University, Nanjing 210031, China)
}

\begin{abstract}
The minimum gripping force applied is expected to prevent objects from mechanical damage when an agricultural robot is applied to handle and manipulate fruits and vegetables. In this research, a sensitive slipping sensor was developed with a piezo resistor to control the griping force of the agricultural robot. Firstly, an output of the slipping sensor was analyzed in a frequency domain by using a short time Fourier transform. Then rules for discriminating slipping signal from the output of a slipping sensor were proposed based on detail coefficients of discrete wavelet transform. Finally, a controller based on adaptive Neuro-Fuzzy inference system was developed to adjust the grasping force of the agricultural robot in real time. The detail coefficients and the normal gripping force were applied as input of the controller, and Fuzzy rules were simplified through subtractive clustering. With a two-finger end-effector of the agricultural robot, the experimental results showed that the slipping signal could be effectively extracted regardless of change in the normal gripping force, and the gripping force had been controlled successfully when grasping tomatoes and apples. This method was a promising way to optimize the gripping force of the agricultural robot grasping the fruits and vegetables.
\end{abstract}

Keywords: agricultural robot, slipping sensor, DWT, gripping force control

DOI: $10.25165 /$ j.ijabe.20181104.3279

Citation: Tian G Z, Zhou J, Gu B X. Slipping detection and control in gripping fruits and vegetables for agricultural robot. Int J Agric \& Biol Eng, 2018; 11(4): 45-51.

\section{Introduction}

In recent years around the world, there has been an upsurge in the number of large, medium, and startup companies showing their interest in development \& improvement of driverless agricultural tractors, robots, equipment's for field management, and dairy milking systems. Moreover, the demand for agricultural robots is actuality driven by the global trends of increase in population, maximum pressure in strain of food supply, availability of farm workers, the challenges and complexities of farm labor, cost for workers, and the automation of the agriculture industry. Therefore, robots are applied for agricultural processes like pruning, weeding, picking and placing, sorting, seeding, spraying and harvesting fruits and vegetables with various kinds of end effectors, a desired grasping force should not only keep them from dropping, but also cause minimum mechanical damage. However, the fruits and vegetables commonly vary in shape, weight and maturity, and their viscoelastic mechanical properties can lead to complex deformation under the action of the grasping force. Thus it is necessary for the agriculture robot to adjust the griping force in real time rather than exerting a predetermined force. Generally, in order to ensure a stable grasp with the minimum griping force, two significant problems must be solved: (i) detection of slippage of the griped objects, especially the detection of 'initial slip'; (ii) determination of the appropriate magnitude of the griping force, the

\section{Received date: 2017-02-25 Accepted date: 2018-02-06}

Biographies: Guangzhao Tian, $\mathrm{PhD}$, Lecturer, research interests: agricultural machinery navigation and control, Email: tgz@njau.edu.cn; Baoxing Gu, PhD, Lecturer, research interests: intelligent agricultural equipment, Email: gbx@njau.edu.cn.

*Correspondence Author: Jun Zhou, PhD, Professor, research interests: intelligent agricultural equipment. College of Engineering, Nanjing Agricultural University, No.40, Dianjiangtai Rd, Pukou District, Nanjing 210031, China. Tel: +86-13914467155, Email: zhoujun@njau.edu.cn. strategy of exerting the force and the control method ${ }^{[1-5]}$.

So far, there are many methods to detect the slippage. Dollar et al. ${ }^{[6,7]}$ investigated an application of piezoelectric polymer contact sensors embedded in compliant robot hand to detect instant fluctuations of signal between fingers and object when a robot hand lifting object in unstructured environments, and output signal of the sensor instantaneously fluctuated with the occurrence of the slippage.

Hasegawa et al. ${ }^{[8,9]}$ developed a slipping sensor with three-layer structure composed of a layer of pressure sensitive conductive rubber and two layers of conductive film. A decline of output of the slipping sensor happened when the slippage occurred. Kai Zhao et al. ${ }^{[10]}$ provided a new method for slip detection based on video processing, which could offer multidimensional sensing information and it is applicable for gripping deformable objects. Cotton et al. ${ }^{[11]}$ applied the piezoelectric ceramic bimorph element as a dynamic force sensor, and the slippage was detected by setting a suitable threshold. Petchartee et al. ${ }^{[12]}$ developed a resistive tactile sensor containing 204 elements, and the partial slippage was predicted by sensing micro vibrations in tangential forces caused by an expansion of the slip regions within the contact area. Shirafuji et al. ${ }^{[13,14]}$ provided a method by which robot hands can be controlled on the basis of previous experience of slippage of objects held by the hand. Lévesque et al. ${ }^{[15]}$ proposed a model-based scooping grasp for the picking of thin objects lying on a flat surface, which were typically elusive to common grippers and grasping strategies. Vatani et al. ${ }^{[16]}$ presented a way of force and slip detection with direct-write compliant tactile sensors using multi-walled carbon nanotube polymer composites.

These detection methods have achieved certain effect, but many problems still exist, and at present there is no real slip sensor included in any commercially available robotic hand. The polyvinylidene fluoride (PVDF) has a high sensitivity to temperature change, and the sensor activity changes by 
approximately $0.5 \% /{ }^{\circ} \mathrm{C}$. It is difficult for the pressure conductive rubber to distinguish between slipping and a change in the normal griping force. The piezoelectric ceramic has a high sensitivity to noises, and a limited velocity in the slipping detection. Due to high resolution and high scanning frequency, the sensor array possesses complex structures and many wires.

In general, strategies applied for the real-time grasping force control can be classified into three groups: (i) Direct and linear-feedback control, the most elementary example is simple on-off control. Proportional and proportional-derivative controllers are used to regulate shear force magnitude when a slip event was detected ${ }^{[17]}$. The difficulty with using linear-feedback controllers in the control of slipping is that their gains are largely derived arbitrarily, and their robustness to parameter variations is unknown. (ii) Force ratio control, which aims to match the coefficient of friction in the object-gripper interface. Koda and Maeno ${ }^{[18]}$ used this approach to modify a demand signal imposed by a human operator in a master-slave system. However, a complex sensor that is able to measure both contact force and shear force, and some characteristics of slip are required. (iii) Model-free control, because object properties such as hardness, mass and friction are uncertain or non-linear and a model cannot be easily developed, model free controllers are appealing in this application, for example Fuzzy logical control, can replace a model with a heuristic rule set, circumventing the need for knowledge of the object properties. Glossas and Aspragathos ${ }^{[19]}$ develop an intelligent control architecture based on Fuzzy Logic, for a robotic grasping system capable of lifting and manipulating an unknown and fragile object. However, the membership functions are defined arbitrarily, so FLCs generally yield suboptimal outputs, which can be partially solved by a neuro-fuzzy logic controller capable of retuning online.

In this study, a new slipping sensor was developed by using piezo resistor, and the corresponding grasping force control was proposed.

\section{Materials and methods}

\subsection{Structure of slipping sensor}

As shown in Figure 1, a two-finger end-effector was equipped on the commercial robotic arm to harvest fruits. And in order to grasp the fruits successfully and prevent them from mechanical damage, a slipping sensor was developed. The slip sensor was made of FSR-406 force sensitive resistance, rubber skin, foam rubber and support plate. FSR-406 is a polymer thick film (PTF) device which exhibits a decrease in resistance when an increasing force exerted on its active surface. The rubber skin with reticulate ridges was applied to cover the sensitive area of the sensor, which could not only protect the force sensitive resistance from damaging, but also increase the friction between the sensor and objects gripped. With processed by transmitter circuit, the output $V_{\mathrm{o}}$ of sensor $(0-5 \mathrm{~V})$ can be calculated as:

$$
V_{O}=\frac{V}{1+R_{F} / R_{M}}
$$

where, $V$ is the source voltage of transmitter $(5 \mathrm{~V}) ; R_{F}$ is the current resistance of FSR; $R_{M}$ is a constant resistance $(21 \mathrm{k} \Omega)$. Therefore, the sensor has a simple structure, large sensitive area, and is easy to be installed.

\subsection{Analysis of output of slipping sensor in frequency domain}

A SCHUNK electrical gripper was applied as the two-finger end-effector, and the slip sensor designed was fixed on one of the

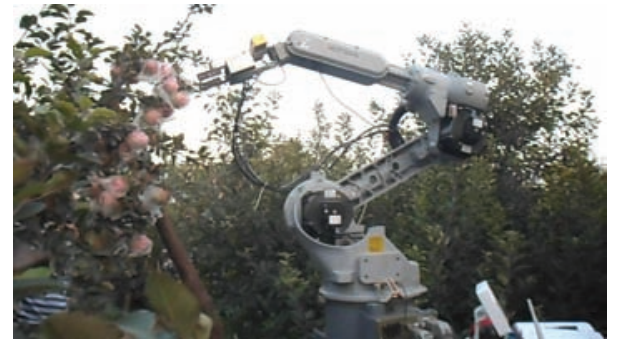

a. Robotic arm with a slipping sensor

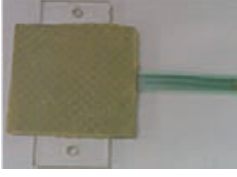

b. Appearance of slipping sensor

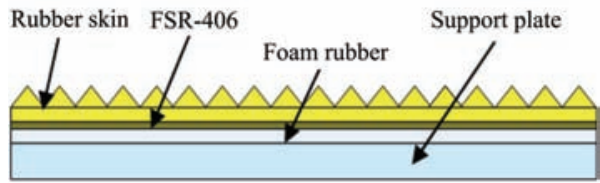

c. Structure of slipping sensor
Figure 1 Slipping sensor

fingers. A wood block was used as the griped object. When drawn by external force, the wood block slipped on the surface of the slip sensor. A touch switch sensor was installed on the experimental stand, and being kept contacting with the wood block before moving between two fingers. The change of contact state will lead to the change in the output voltage of touch sensor, which can indicate the time when the slip happens between the wood block and the finger in the experiment. On the other finger a force sensor was equipped to measure the griping normal force. The signals of these sensors were sampled by a PCI-1710 multi-channel data acquisition card, and processed on an industrial computer.

In the experiment, the wood block was placed between the two fingers firstly, and the normal gripping force was adjusted to be $2 \mathrm{~N}$. Then the wood block was drawn to slip between the two fingers with speeds ranging from $5 \mathrm{~mm} / \mathrm{s}$ to $30 \mathrm{~mm} / \mathrm{s}$. The typical output of the slip sensor is shown in Figure 2. The dotted line illustrates that the touch sensor output decreased from $4.3 \mathrm{~V}$ to $0.67 \mathrm{~V}$, which indicates the separation between the wood block and the touch sensor, that is, the wood block began to slip at $2.6 \mathrm{~s}$. The solid curve represents the output of slip sensor, and the complex and violent fluctuations occurred from $2 \mathrm{~s}$, and peaked at $2.6 \mathrm{~s}$, finally stabilize after $4 \mathrm{~s}$. In contrast to the output of touch sensor the shadow area (Figure 2) can represent the static friction process occurring before the visible slipping of wood block. So the fluctuations of signal between $2.6 \mathrm{~s}$ and $4 \mathrm{~s}$ can indicate the transition from static to kinetic friction. Moreover, these fluctuations of output of slip sensor in the static friction area show that it is possible to detect 'Initial slip' immediately before the visible slipping of griped object happens.

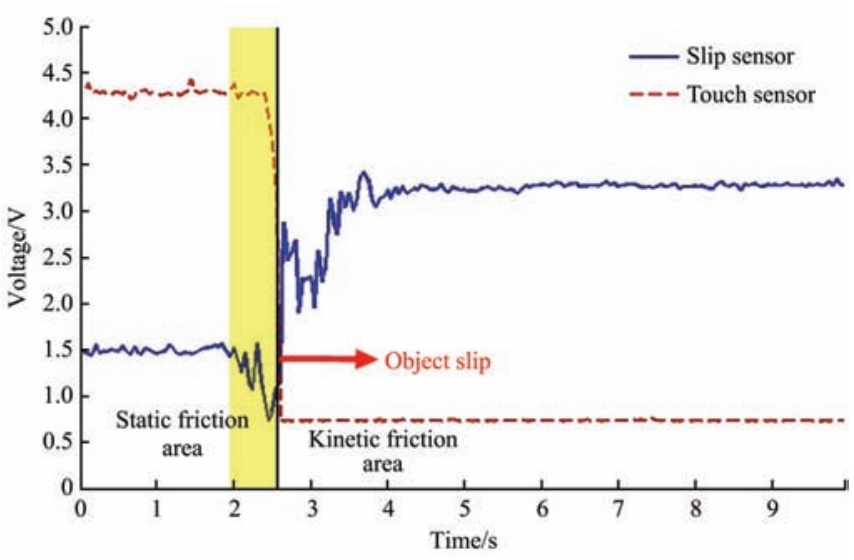

Figure 2 Typical output of the slipping sensor 
Slipping information is the basis for adjusting the grasping force of the robotic end-effector and realizing the soft grasping which can prevent grasped objects from mechanical damage. In order to find suitable algorithm that can effectively extract the slip information from the output of slip sensor, the short-time Fourier transform (STFT) of the output of slip sensor was performed to observe its time-frequency characteristics (Figure 3). The fluctuations of the output signal of the slip sensor are small at the initial slide of the wood block until $3.2 \mathrm{~s}$, and the signal intensities with high-frequencies are small. However, during time interval from $3.2 \mathrm{~s}$ to $5.2 \mathrm{~s}$, the signal intensities with high-frequencies increase immediately although the visible moving of wood block has not happened yet at this stage, and friction between the wood block and the surface of slip sensor changes from static to kinetic state. After $5.2 \mathrm{~s}$, the wood block began sliding, the signal powers with high-frequencies decreases also.

The components with high-frequencies in the output of the slip sensor are generated by characteristics of polymer thick film material primarily composed of silicon rubber with carbon particles uniformly distributed. When the friction is exerted on the material surface, the carbon particles redistribute and a new conduction route forms. This leads to a change in electrical resistance, which causes the voltage change in the output of the slip sensor ${ }^{[20]}$. Therefore, the gripping process of the robotic end-effector and the slipping information of the objects grasped can be obtained through detecting the frequency characteristics of the output signals of this kind of slip sensor in a serial of short-time intervals.
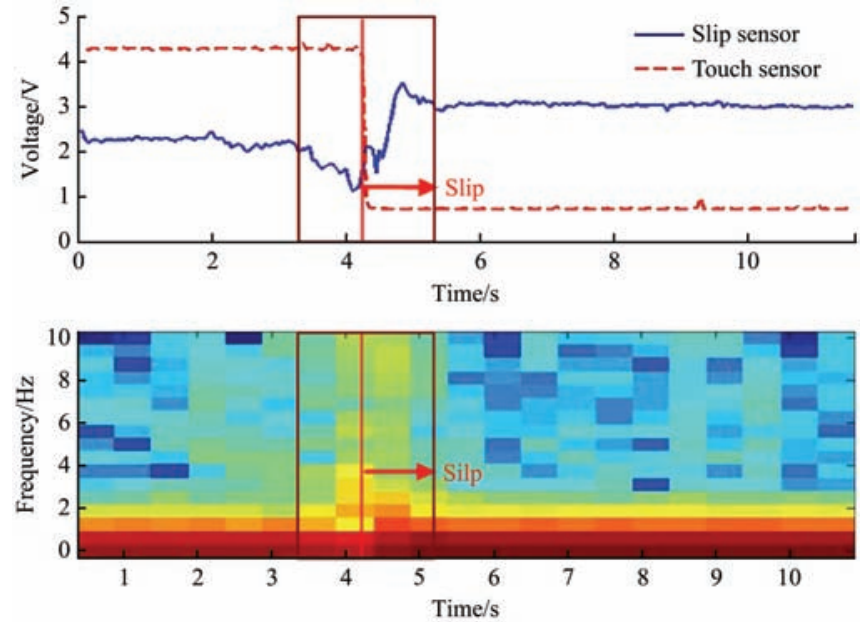

Figure 3 STFT of the output of slipping sensor

\subsection{Detecting slippage based on discrete wavelet transform}

A discrete wavelet transform (DWT) is a wavelet transform for which the wavelets are discretely sampled and can capture both frequency and location information. The detail coefficients of DWT can reflect the components with high frequencies in the signal. Due to its time saving the wavelet of Haar was used to achieve the DWT, and the first-order detail coefficients were calculated.

As loading and unloading of normal grasping force can also cause a change in the output of slip sensor, it is necessary to separate the slip signal from the change in the normal grasping force signal. Figures 4 and 5 reveal the DWT of the output of slip sensor when slipping happened with constant normal grasping force and when the normal grasping force changed without any slipping, respectively. As a result, their amplitudes of detail coefficients of DWT are almost the same. Therefore, it is impossible to distinguish the situation between slipping of grasped objects and the change in the normal grasping force only by comparing the absolute value of detail coefficients.
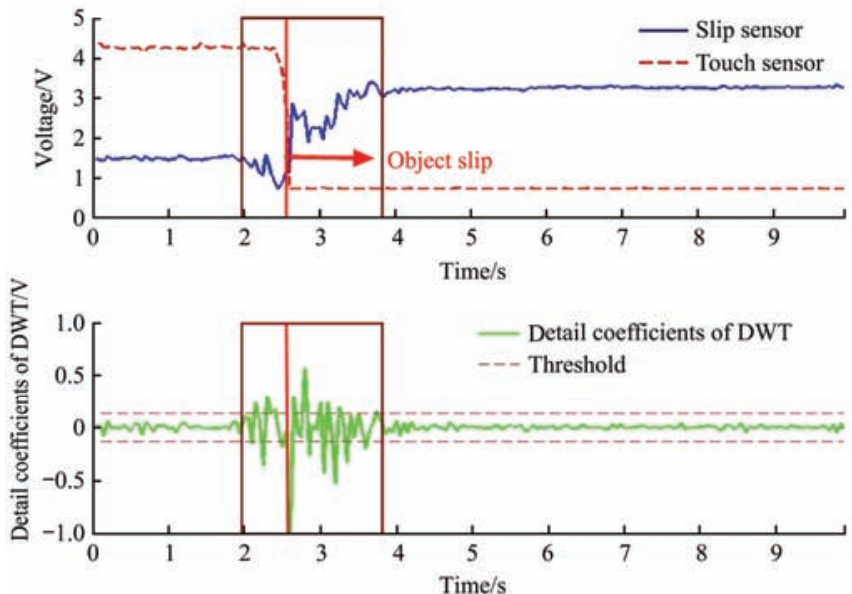

Figure 4 Slip sensor data and corresponding DWT when grasped object slipping
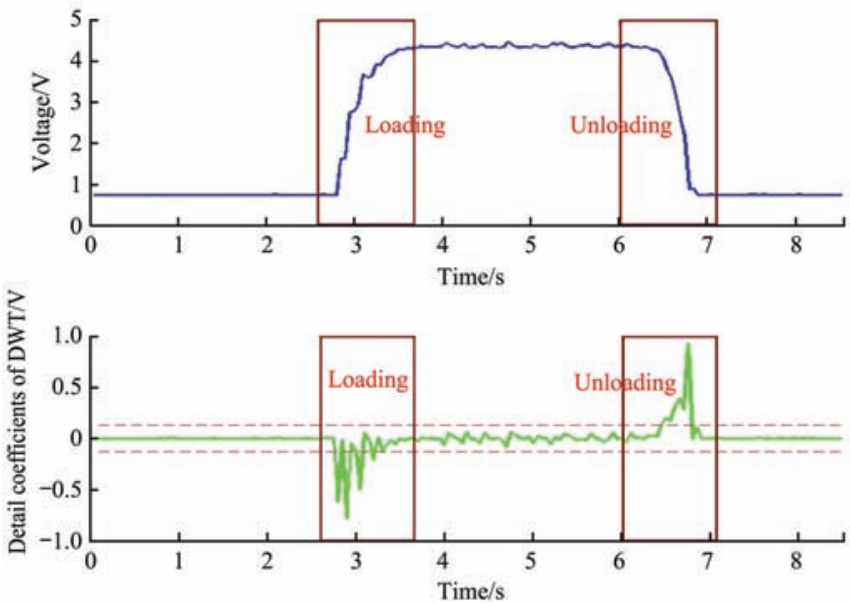

Figure 5 Slip sensor data and corresponding DWT when normal grasping force changing

However, from Figure 5 it can be found that the most detail coefficients were negative for loading of normal grasping force, whereas they became positive for unloading, which is obviously different with the results in Figure 4 where not only their amplitudes are high when the slipping happened, but also the detail coefficients contain both the negative and positive values.

Based on the analysis above and the results of experiments, the threshold rules were proposed to detect the slipping signal: i) when increasing of normal grasping force, as long as the detail coefficients of DWT were more than 0.13 ; ii) when decreasing of normal grasping force, if the detail coefficients of DWT were less than -0.13 ; iii) when normal grasping force was constant, as long as the absolute values of detail coefficients of DWT were greater than 0.13. As shown in Figure 4, these detecting rules can be satisfied in the static friction stage, which means that the slipping information can be obtained before the visible moving of grasped objects happens. Moreover, these detecting rules are also robust to noise due to the threshold is set to be more than zero.

\subsection{Control of gripping force}

The ultimate purpose of detecting the slipping based on the detail coefficients of DWT of the output of slip sensor was to optimize and gain the minimum grasping force of the robotic 
end-effector, which can prevent the grasped objects from damaging. Therefore, the control of grasping force according to feedback of the slip sensor will be discussed firstly in this section.

Because physical properties of fruits and vegetables are very complex, a method of control of grasping force based on adaptive neuro-fuzzy inference system (ANFIS) was developed, in which mathematical models of grasped objects are unnecessary. ANFIS is a kind of neural network based on Takagi-Sugeno fuzzy inference system. Since it integrates both neural networks and fuzzy logic principles, it has potential to capture the benefits of both in a single framework.

When the absolute value of detail coefficients was small, we could consider the slipping degree was small, and vice versa. However, the mathematical relationship between them was complicated. Moreover, according to the results of previous experiments (Table 1), the normal grasping force has influence on the detail coefficients. So it is obviously inapplicable to use the detail coefficient as the only input of ANFIS. And here both the current normal griping force $F$ and the detail coefficients $d w t$ of DWT were chosen as the inputs of the ANFIS controller to adjust the grasping force when slipping was detected, the increment $\Delta d$ of distance between the two fingers of end effector was taken as the output of ANFIS controller.

The IF-THEN rules based on Fuzzy Logic were indispensable components in the ANFIS controller. Initially an input-output data set was collected from successful grasp and lifting of a wide range of objects. This input-output data set was used to train the controller based on subtractive clustering. And each clustering center would correspond to one rule. As a result, the Fuzzy rules were simplified and the controller was considered more flexible. The criteria for a successful grasping are defined as: (i) the dropping of the grasped object does not happen; (ii) the maximum force $F_{\max }$ of grasping is less than 1.2 times the minimum grasping force. The way to measure the minimum grasping force is as follows: to decrease the grasping force gradually until the slipping signal of grasped object appears, and at this moment record the value of griping force sensor in the finger.

At last, a hybrid algorithm consisting of a gradient descent algorithm and least square algorithm was implemented to tune the antecedent parameters and consequent part of the ANFIS controller.

The final structure of ANFIS controller is shown in Figure 6. Based on the data of slip sensor designed and the robotic end-effector used in the experiments, four Fuzzy rules were achieved as follows.

Rule 1: if $F$ is $F_{1}=g(F ; 0.308,2.275)$ and $d w t$ is $d w t_{1}=g(d w t ; 0.1687$, $0.2599)$

then $\Delta d_{1}=1.218 F+17.32 d w t+1.078$

Rule 2: if $F$ is $F_{2}=g(F ; 0.3097,2.705)$ and $d w t$ is $d w t_{2}=g(d w t$; $0.1733,0.3882)$

then $\Delta d_{2}=1.932 F+1.705 d w t-6.457$

Rule 3: if $F$ is $F_{3}=g(F ; 0.3097,0.8942)$ and $d w t$ is $d w t_{3}=g(d w t$; $0.1814,0.3441)$

then $\Delta d_{3}=-0.1319 F+0.2646 d w t+0.745$

Rule 4: if $F$ is $F_{4}=g(F ; 0.3118,2.151)$ and $d w t$ is $d w t_{4}=g(d w t$; $0.1604,0.2769$ )

Then $\Delta d_{4}=-7.352 F-11.75 d w t+12.42$

where, $g(x ; \sigma, c)$ is Gaussian membership function of Fuzzy logic, and $x$ denotes viable, $\sigma$ denotes standard deviation, $c$ denotes the mean value, that is the clustering center.

When the input is $(F, d w t)$, the output of the ANFIS controller:

$$
\Delta d=\frac{\sum_{i=1}^{4}\left|F_{i} d w t_{i}\right| \cdot \Delta d_{i}}{\sum_{i=1}^{4}\left|F_{i} d w t_{i}\right|}
$$

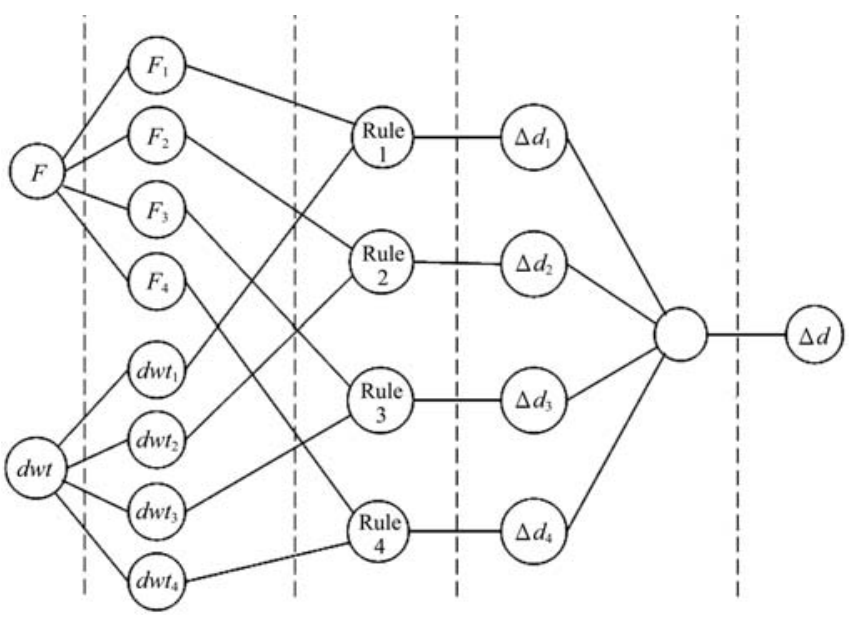

Figure 6 Structure of adaptive neuro-fuzzy inference system

\section{Results and discussion}

\subsection{Experimental results of slipping detection}

In order to evaluate performance of detecting rules developed based on the detail coefficients of DWT, three groups of experiments were carried out: i) the normal grasping force were set to be $1.5 \mathrm{~N}, 2 \mathrm{~N}, 3 \mathrm{~N}$ and $4 \mathrm{~N}$ respectively, and sliding speed of the wood block over the surface of slip sensor was $15 \mathrm{~mm} / \mathrm{s}$; ii) the surface materials of grasped objects were chosen to be wood, plastic, cloth, and paper respectively, and sliding speed still was $15 \mathrm{~mm} / \mathrm{s}$ with the $2 \mathrm{~N}$ normal grasping force; 3 ) the sliding speeds were set to be $8 \mathrm{~mm} / \mathrm{s}, 15 \mathrm{~mm} / \mathrm{s}, 25 \mathrm{~mm} / \mathrm{s}$ respectively, and the normal grasping force of wood block was maintained to be $2 \mathrm{~N}$.

The results of each experiment were similar with the ones presented in the Figure 4. And peak values of the detail coefficients in the DWT of the output of slip sensor before the actual moving of the grasped objects were depicted in Tables 1-3, respectively.

Table 1 Peak values of detail coefficients of DWT before actual slipping with different normal grasping forces

\begin{tabular}{cc}
\hline Normal grasping force/ $\mathrm{N}$ & Peak values of detail coefficients \\
\hline 1.5 & -0.6803 \\
2 & -0.5577 \\
3 & -0.651 \\
4 & -0.1658 \\
\hline
\end{tabular}

Table 2 Peak values of detail coefficients of DWT before actual slipping with different surface materials of grasped objects

\begin{tabular}{cc}
\hline Surface material & Peak value of detail coefficients \\
\hline Wood & -0.5215 \\
Plastic & 0.7114 \\
Cloth & 0.2728 \\
Paper & -0.2331 \\
\hline
\end{tabular}

Table 3 Peak values of detail coefficients of DWT before actual slipping with different sliding speeds

\begin{tabular}{cc}
\hline Sliding speed $/ \mathrm{mm} \cdot \mathrm{s}^{-1}$ & Peak value of detail coefficients \\
\hline 8 & 0.2954 \\
15 & 0.9843 \\
25 & -0.3574 \\
\hline
\end{tabular}


In Table 1, when the normal grasping force is $4 \mathrm{~N}$ the corresponding detail coefficient is less than that when the normal grasping force being $1.5 \mathrm{~N}, 2 \mathrm{~N}$ and $3 \mathrm{~N}$, respectively. This was likely because the greater normal grasping force can lead to output saturation of slip sensor, which weakens the components with high frequencies in the output of slip sensor. In Table 2, due to the smooth surface of the paper the corresponding detail coefficient is smallest, and the same reason for one with the cloth material. Finally, in Table 3, for the sliding speed of $15 \mathrm{~mm} / \mathrm{s}$ and $25 \mathrm{~mm} / \mathrm{s}$, their corresponding detail coefficients become greater than that when sliding speed is $8 \mathrm{~mm} / \mathrm{s}$. The reason for this was that the slower the sliding speed, the smaller vibration of friction between the rubber skin and the grasped objects can be caused. And in the case of sliding tends to zero, the absolute values of the detail coefficient will decrease toward zero, which is just the desired situation.

However, although the test conditions such as normal grasping forces, the surface material properties and the sliding speeds were set at different levels, the slipping detection rules proposed above can be satisfied totally, which implied the slipping can be measured successfully before the visible slipping of grasped objects occurred, that is, the initial slip information can be detected effectively.

\subsection{Experimental results of gripping force control}

As shown in Figure 7, in order to test the effectiveness of ANFIS controller, the experiments for adjusting the grasping force were carried out by using a two-finger end-effector. The slip sensor was installed in one finger, and the grasping force sensor was installed in the other finger.

Firstly, an empty plastic cup was held by the robotic end-effector, and the coins were added into the cup one by one. So the total weight of cup increased gradually. When the holding force of end-effector was not enough to overcome the total gravity the cup would slip between the two fingers. This slipping would be measured by the slip sensor, and the ANFIS controller would be activated to adjust the gripping force to prevent the cup from slipping.
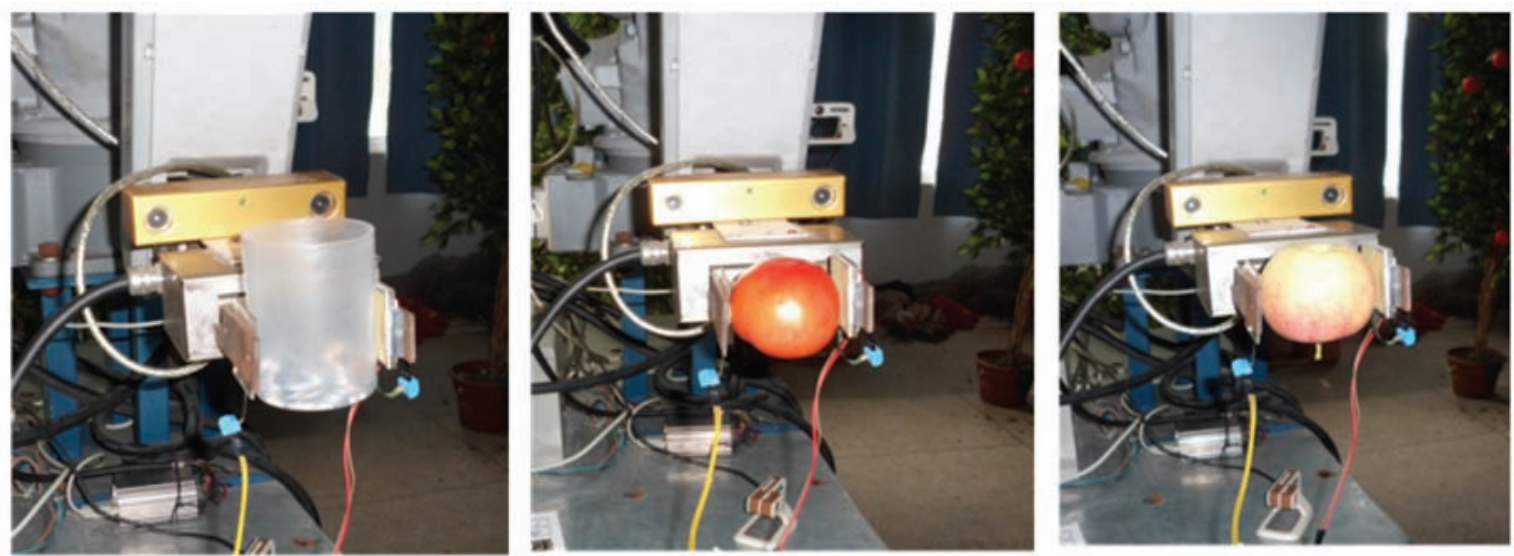

Figure 7 Experiment of griping force control

Secondly, tomatoes and apples were randomly selected and placed between the two fingers of the end-effector manually. After contacting between the fingers and grasped objects the grasping force would be controlled according to the slipping detection of the slip sensor by the ANFIS controller.

The results of grasping plastic cup are illustrated in Figure 8. The upper sub-graph shows the output of slip sensor, the middle sub-graph shows the griping force applied by the end-effector, and the lower sub-graph shows the detail coefficients of DWT of the slip sensor output. When the weight of the plastic cup increased at $0.9 \mathrm{~s}, 1.8 \mathrm{~s}, 2.9 \mathrm{~s}$ and $3.7 \mathrm{~s}$, the detail coefficients of DWT became greater than the threshold 0.13 , which indicated that the slipping of cup had happened. So the ANFIS controller of grasping force of end-effector worked immediately to prevent the cup from slipping based on the slipping data detected. In one experiment, the total displacement of the cup slipping was less than $2 \mathrm{~mm}$ measured according to the line marked on the cup.

The typical results of grasping tomatoes and apples are shown in Figure 9 and Figure 10, respectively. So the tested samples were selected randomly, and weight of the tomato is $143 \mathrm{~g}$ and apple is $166 \mathrm{~g}$. Their minimum griping force was $2.0 \mathrm{~N}$ and $2.5 \mathrm{~N}$ respectively.

The tomato was placed between two fingers of end-effector by hand firstly (Figure 9), and after the finger contacted with the tomato at $3.2 \mathrm{~s}$, the grasping force increased to $1.2 \mathrm{~N}$ and was kept till we moved the hand away at $4.8 \mathrm{~s}$ completely. Then the tomato began to slip because the griping force was smaller than the tomato's minimum griping force $2.0 \mathrm{~N}$. It could be found that the detail coefficient of DWT was greater than 0.13 at this time. Therefore, the ANFIS controller adjusted the grasping force to $2.1 \mathrm{~N}$ automatically which was slightly greater than the minimum force. However, the grasping force slightly decreased because of the deformation of the tomato, and at $8.2 \mathrm{~s}$, the slip sensor detected the initial slip of the tomato again and the grasping force increased once more by ANFIS controller autonomously.
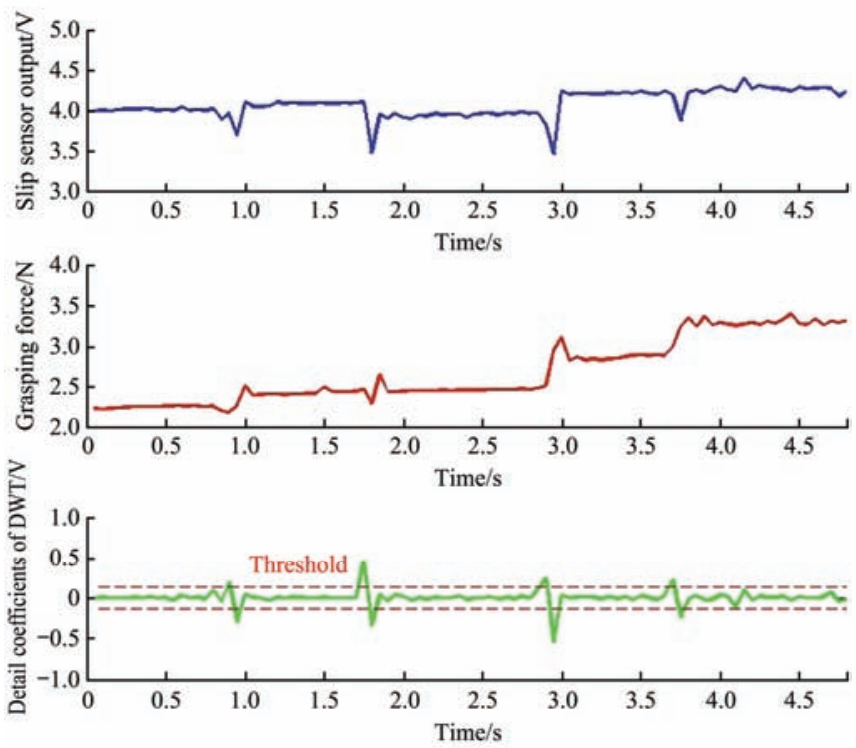

Figure 8 Results of grasping plastic cup with increasing weight 


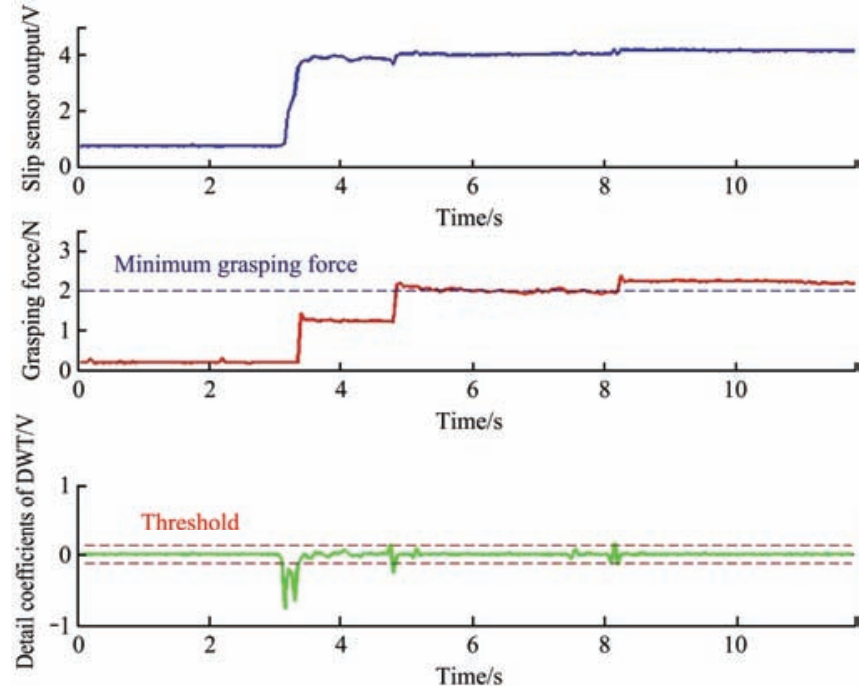

Figure 9 Control of griping force for tomato
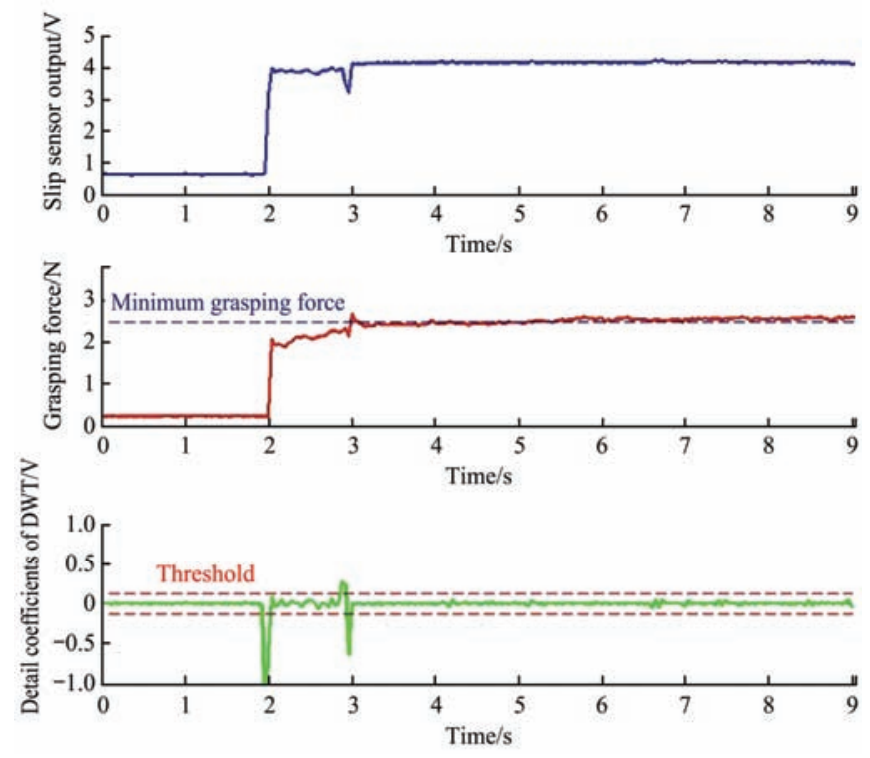

Figure 10 Control of griping force for apple

The typical process of grasping apple is shown in Figure 10, which is similar to that of the tomato. The detail coefficient of DWT was greater than threshold 0.13 at $2.9 \mathrm{~s}$ when hand moved away. So the grasping force increased, and the peak force reached to $2.7 \mathrm{~N}$. Because the deformation of apple is negligible under the minimum gripping force the second slipping of the apple has not been found.

In these experiments, all tested objects had been grasped without dropping. Moreover, the grasping force did not appear the big overshoot, which could effectively prevent the fruit and vegetable from mechanical damage.

\section{Conclusions}

When the robots are applied to manipulate the fruits and vegetables, minimum griping force is desired, which can reduce the mechanical damage in the grasping process. Thus in this research, a new and sensitive slip sensor was developed by exploiting the characteristics of force sensitive resistor. The output of slip sensor was analyzed in the frequency domain with the STFT, and components with high frequencies were analyzed carefully. These components with high frequencies were further processed with the DWT. And based on the detail coefficients of DWT the rules of detecting the slipping of the grasped objects were introduced.

With the detection of slipping and the measuring of the normal grasping force, the ANFIS controller was developed to adjust the gripping force of the robotic end-effector in real time, and the subtractive clustering was applied to simplify the fuzzy logical rules effectively with the two parallel fingers robotic end-effector. Hence the results of experiments demonstrated the effectiveness of the method for detecting the slipping and adjusting the grasping force.

\section{Acknowledgments}

This work was supported by the Key Research and Development Program of Jiangsu (Grant No.BE2017370), the National Natural Science Foundation of China (Grant No.31471419), and the Natural Science Funds of Jiangsu (Grant No.BK20140729).

\section{[References]}

[1] Teshigawara S, Tsutsumi T, Shimizu S, Suzuki Y. Highly sensitive sensor for detection of initial slip and its application in a multi-fingered robot hand. IEEE International Conference on Robotics and Automation, Shanghai, China, 2011; 19(6): 1097-1102.

[2] Damian D D, Martinez H, Dermitzakis K, Hernandez-Arieta A. Artificial ridged skin for slippage speed detection in prosthetic hand applications. IEEE/RSJ International Conference on Intelligent Robots and Systems, Taipei, Taiwan, 2010; 25(1): 904-909.

[3] Shang Z D, Wang Q Y, Han J H. Sliding sensor and soft grasping of electron hydraulic servo manipulator. IEEE International Conference on Mechatronics and Automation, Luoyang, China, 2006; pp.1459-1464.

[4] O’Toole M, Bouazza-Marouf K, Kerr D, Vloeberghs M. Robust contact force controller for slip prevention in a robotic gripper. Proceedings of the Institution of Mechanical Engineers, Part I: Journal of Systems \& Control Engineering, 2010; 224(3): 275-288.

[5] Sanchez J, Schneider S, Hochgeschwender N, Kraetzschmar G K, Plöger P G. Context-based adaptation of in-hand slip detection for service robots. IFAC Papers On Line, 2016; 49(15): 266-271.

[6] Dollar A M, Jentoft L P, Gao J H, Howe R D. Contact sensing and grasping performance of compliant hands. Autonomous Robots, 2010; 28(1): 65-75.

[7] Dollar A M, Cho K J, Fearing R S, Park Y L. Special issue: fabrication of fully integrated robotic mechanisms. Journal of Mechanisms \& Robotics, 2015; 142(S266): 419-428.

[8] Hasegawa H, Mizoguchi Y, Tadakuma K, Ming A. Development of intelligent robot hand using proximity, contact and slip sensing. IEEE International Conference on Robotics and Automation, Anchorage, AK, USA, 2010; 46(1):777-784.

[9] Gunji D, Mizoguch Y, Teshigawara S, Ming A, Namiki A, Ishikawa M, et al. Grasping force control of multi-fingered robot hand based on slip detection sing tactile sensor. JRSJ, 2010; 25: 2605-2610.

[10] Zhao K, Li X, Lu C, Lu G, Wang Y. Video-based slip sensor for multidimensional information detecting in deformable object grasp. Robotics \& Autonomous Systems, 2017; 91: 71-82.

[11] Cotton D P J, Chappell P H, Cranny A, White N M, Beeby S P. A novel thick-film piezoelectric slip sensor for a prosthetic hand. IEEE Sensors Journal, 2007; 7(5): 752-761.

[12] Petchartee S, Monkman G. Slip Prediction through Tactile Sensing. Sensor and Transducers Journal (CD-ROM), 2008; 90: 310-324.

[13] Shirafuji S, Hosoda K. Detection and prevention of slip using sensors with different properties embedded in elastic artificial skin on the basis of previous experience. International Conference on Advanced Robotics, Tallinn, Estonia, 2011; 62(1): 459-464.

[14] Shirafuji S, Ikemoto S, Hosoda K. Trajectory control strategy for anthropomorphic robotic finger. Conference on Biomimetic and Biohybrid Systems, Milan, Italy, 2014; 8608(3): 284-295.

[15] Lévesque F, Sauvet B, Cardou P, Gosselin C. A model-based scooping 
grasp for the autonomous picking of unknown objects with a two-fingered gripper. Robotics \& Autonomous Systems, 2018; 106: 14-25.

[16] Vatani M, Engeberg E D, Choi J W. Force and slip detection with direct-write compliant tactile sensors using multi-walled carbon nanotube polymer composites. Sensors \& Actuators A: Physical, 2013; 195(2): 90-97.

[17] Engeberg E D, Meek S G. Adaptive object slip prevention for prosthetic hands through proportional-derivative shear force feedback. IEEE/RSJ International Conference on Intelligent Robots and Systems, Nice, France, 2016; pp.1940-1945
[18] Koda Y, Maeno T. Grasping force control in master-slave system with partial slip sensor. IEEE/RSJ International Conference on Intelligent Robots and Systems, Beijing, China, 2006; 740: 4641-4646.

[19] Glossas N I, Aspragathos N A. A cluster based fuzzy controller for grasp and lift fragile objects. 18th Mediterranean Conference on Control \& Automation, Marrakech, Morocco, 2010; 20(1): 1139-1144.

[20] Teshigawara S, Tadakuma K, Ming A, Ishikawa M. Development of high-sensitivity slip sensor using special characteristics of pressure conductive rubber. IEEE International Conference on Robotics and Automation, Kobe, Japan, 2009; pp.3289-3294. 